\title{
BMJ Open Provision of medical assistance in dying: a scoping review
}

\author{
Max Zworth (D , , ${ }^{1}$ Carol Saleh, ${ }^{2}$ Ian Ball, ${ }^{3}$ Gaelen Kalles, ${ }^{4}$ Anatoli Chkaroubo, ${ }^{4}$ \\ Mike Kekewich, ${ }^{5}$ Paul Q Miller, ${ }^{4,6}$ Marianne Dees, ${ }^{7}$ Andrea Frolic, ${ }^{4}$ \\ Simon Oczkowski (iD 4,8
}

To cite: Zworth M, Saleh C, Ball I, et al. Provision of medical assistance in dying: a scoping review. BMJ Open 2020;10:e036054. doi:10.1136/ bmjopen-2019-036054

- Prepublication history and additional material for this paper are available online. To view these files, please visit the journal online (http://dx.doi. org/10.1136/bmjopen-2019036054).

Received 11 December 2019 Revised 25 May 2020 Accepted 29 May 2020

Check for updates

(c) Author(s) (or their employer(s)) 2020. Re-use permitted under CC BY-NC. No commercial re-use. See rights and permissions. Published by BMJ.

For numbered affiliations see end of article.

Correspondence to

Dr Max Zworth;

max.zworth@medportal.ca

\section{ABSTRACT}

Objectives The purpose of this study is to map the characteristics of the existing medical literature describing the medications, settings, participants and outcomes of medical assistance in dying (MAID) in order to identify knowledge gaps and areas for future research.

Design Scoping review.

Search strategy We searched electronic databases (MEDLINE, EMBASE, PsychINFO, CINAHL and CENTRAL), clinical trial registries, conference abstracts and professional guidelines from jurisdictions where MAID is legal, up to February 2020. Eligible report types included technical summaries, institutional policies, practice surveys, practice guidelines and clinical studies that describe MAID provision in adults who have provided informed consent for MAID.

Results 163 articles published between 1989 and 2020 met eligibility criteria. 75 studies described details for MAID administered by intravenous medications and 50 studies provided data on oral medications. In intravenous protocols, MAID was most commonly administered using a barbiturate (34/163) or propofol (22/163) followed by a neuromuscular blocker. Oral protocols most often used barbiturates alone (37/163) or in conjunction with an opioid medication (7/163) and often recommended using a prokinetic agent prior to lethal drug ingestion. Complications included prolonged duration of the dying process, difficulty in obtaining intravenous access and difficulty in swallowing oral agents. Most commonly, the role of physicians was prescribing (83/163) and administering medications (75/163). Nurses' roles included administering medications (17/163) and supporting the patient (16/163) or family (13/163). The role of families involved providing support to the patient (17/163) and bringing medications from the pharmacy for selfadministration (4/163).

Conclusions We identified several trends in MAID provision including common medications and doses for oral and parenteral administration, roles of healthcare professionals and families, and complications that may cause patient, family and provider distress. Future research should aim to identify the medications, dosages, and administration techniques and procedures that produce the most predictable outcomes and mitigate distress for those involved.

\section{INTRODUCTION}

In 2016, the Canadian government passed Bill C-14, which decriminalised medical assistance

\section{Strengths and limitations of this study}

- We conducted a scoping review of medical assistance in dying (MAID) provision using a very broad and inclusive search strategy and a prepublished protocol.

- Screening was performed in duplicate by two investigators at both the title/abstract and full-text levels.

- We describe a wide variety of methods for providing MAID, though few reports described the number of times the protocol has been used.

- The reports we found did not generally link data between medications, locations, providers and outcomes, making it difficult to determine which medications or combinations of medications are most effective and result in the fewest complications.

- Our study is limited by its emphasis on Canadian practice, which is likely due both to most authors being Canadian and the more standardised approaches to MAID provision in European countries compared with North America.

in dying (MAID) for capable patients with intolerable suffering for whom death was 'reasonably foreseeable'. 'As of October 2018, there have been over 6749 medically assisted deaths in Canada, and MAID accounted for approximately $1.12 \%$ of all deaths in Canada in the first 10 months of $2018 .^{2}$ Bill C-14 legislated eligibility criteria under which patients could receive MAID, but provided no guidance on the clinical aspects of providing aid in dying. Critical clinical issues remain unaddressed, such as which pharmaceuticals, doses and routes of administration should be used to cause death; the roles, scope of practice and training requirements for healthcare professionals; the optimal locations for MAID (community, institutional settings or in dedicated centres); and ways to support patients and their families around the time of an assisted death. Several other jurisdictions currently permit MAID in the form of assisted suicide (Switzerland and the American states of Oregon, Montana, Washington, California, Colorado, Vermont, Washington, DC, 
New Jersey, Maine and Hawaii), euthanasia (Columbia) or both (Belgium, the Netherlands and Luxembourg). ${ }^{3}$ While states such as Oregon maintain detailed records for all cases of MAID, ${ }^{4}$ there are few centralised protocols for MAID provision in these settings, ${ }^{5}$ and there remains little readily available evidence to assist Canadian clinicians and organisations in addressing these questions. Thus, Canadian healthcare providers and organisations had to rapidly develop policies and practices for the assessment and provision of MAID in anticipation of this legislative change. Some provinces (such as Alberta and Manitoba) have developed highly centralised care coordination services, whereas others (such as Ontario) have adopted a hands-off approach, allowing individual clinicians and healthcare organisations to develop local policies and protocols for MAID. As a result, there is significant variation in how MAID is practised across Canada.

This is worrisome, as data from other countries suggest that clinical problems with MAID care are common, including poor communication between healthcare providers and patients, inconsistent application of eligibility criteria, unequal access and technical problems with medication administration. ${ }^{6-10}$ Though new federal reporting requirements for MAID took effect in 2018, the collected data are descriptive and not intended to evaluate the quality or consistency of MAID provision. ${ }^{11}$ Although an abundance of literature has emerged in recent years discussing ethical questions around MAID and the experiences of those involved in the MAID process, there is relatively sparse literature addressing the medical aspects of providing aid in dying. Thus, we conducted a scoping review on MAID provision in all jurisdictions where medically assisted dying is practised, with two primary objectives:

1. To describe the range and scope of the existing medical literature on the provision of MAID.

2. To summarise reports of the technical aspects of MAID provision, including pharmaceuticals and procedures; location of provision; the role and scope of involved healthcare professionals; role of patients' families and descriptions of adverse events.

\section{METHODS}

\section{Protocol and registration}

The methods of this scoping review are based on those described in the Joanna Briggs Institute Reviewers Manual $^{12}$ and are described in detail in a previously published study protocol. ${ }^{13}$

\section{Eligibility criteria}

Eligible sources included technical reports, institutional policies, practice surveys, clinical practice guidelines and clinical studies. Opinion pieces/letters were excluded, as were reports solely describing the assessment of patient eligibility for MAID. No restrictions were imposed based on methodological quality, study location, language or publication date. We included reports referring to adult (age $>18$ years) patients who provided informed consent for MAID in the form of either assisted suicide (selfadministered lethal medications) or voluntary euthanasia (lethal medications administered by another person). We included reports describing the provision of MAID using any medication delivery method, in institutions and residences, which involved a healthcare professional such as a physician, nurse or pharmacist. We excluded reports describing other end-of-life practices, including withholding or withdrawing life-sustaining treatment; palliative sedation or unintentional hastening of death via medications for symptom management, unless such reports also included separate descriptions of MAID. Studies in which patients received euthanasia without having provided informed consent (eg, capital punishment) were excluded (table 1).

\section{Information sources and searches}

Briefly, we conducted systematic searches of multiple online databases, including MEDLINE, EMBASE, CINAHL, CENTRAL and PsycINFO from database inception to February 2020 for the concept of MAID ('[medical]) aid [assistance] in dying', 'euthanasia', 'assisted suicide', '[physician] assisted dying', [physician] assisted death', 'end of life choice') and the concept of medication administration ('practice patterns', 'drug administration', 'medication management', 'drug utilization', 'drug therapy'). Complete search details are available in the online supplementary file 1 . We also conducted extensive grey literature searches, including clinical trial databases, conference abstracts from palliative care conferences, technical reports of MAID protocols and institutional policies for MAID until June 2018. Finally, we contacted professional groups and government agencies that monitor and regulate healthcare to obtain protocols and reports describing the provision of MAID.

\section{Selection of sources of evidence}

Report eligibility was determined first by title and abstract screening and second by full-text screening. After pilot testing the screening and eligibility forms on the first 100 abstracts and 10 full-text papers, two investigators (CS and SO) independently reviewed each report's eligibility for inclusion in the review. During the course of the review, no changes were made to the inclusion or exclusion criteria.

\section{Data charting process}

We conducted calibration exercises on the first five eligible studies to pilot test the extraction form and ensure consistent data collection. Two investigators (MZ and CS) then independently extracted data using structured forms divided into three major concepts: report characteristics, methods of MAID provision and MAID outcomes (online supplementary file 2). The data collection form was not modified throughout the extraction process. As our study's objectives were descriptive, we did 
Table 1 Inclusion and exclusion criteria

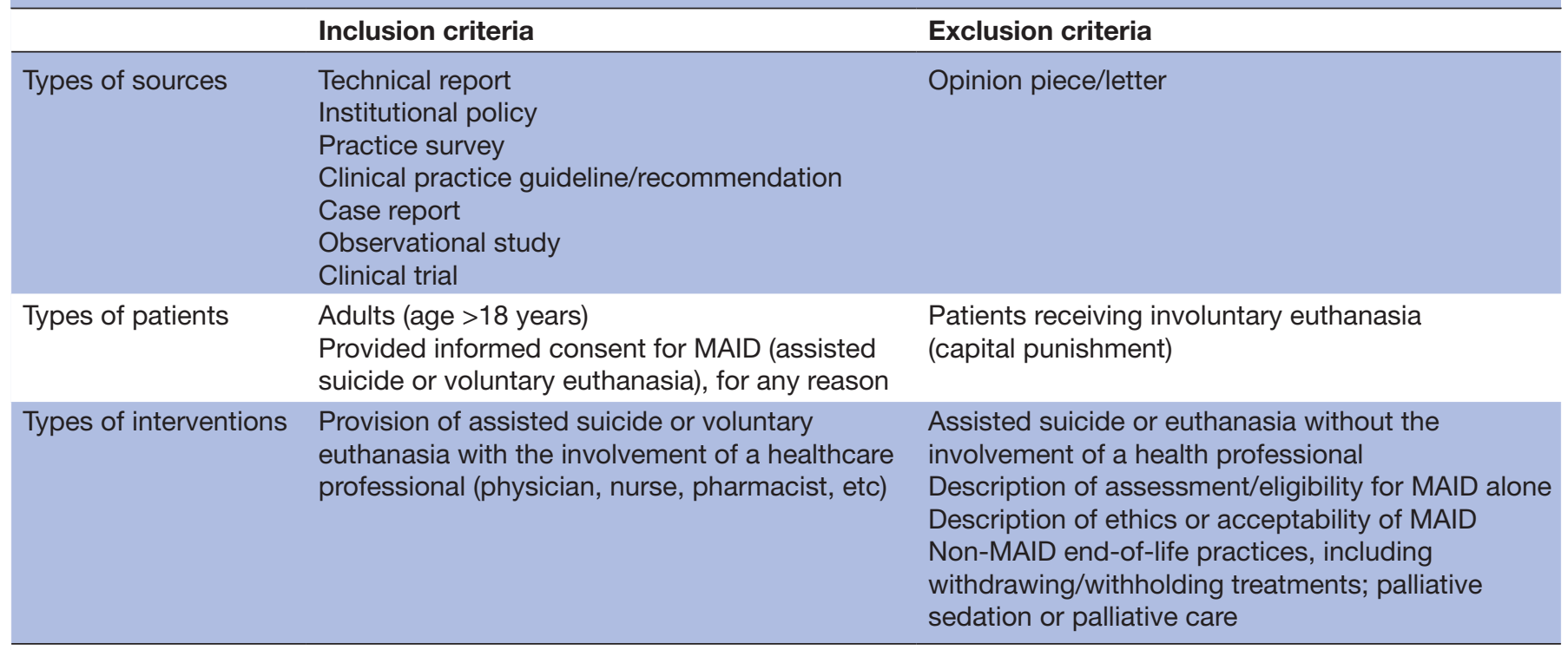

MAID, medical assistance in dying.

not conduct a critical appraisal of the individual studies we retrieved.

\section{Synthesis of results}

Data were organised according to the three major concepts listed above (report characteristics, MAID provision and MAID outcomes). Univariate descriptive statistics were computed for report characteristics, including year of publication, report type and report purpose, in order to provide an overview of the scope and content of the existing literature on MAID. Descriptive statistics (frequency and proportion of studies) were also calculated for categorical data regarding MAID provision, including medications and dosages used in intravenous and oral protocols, order of medication administration and MAID locations. Non-categorical information about MAID provision such as the roles ascribed to various health professionals and safety checks was compiled into a list format, and a team of three investigators extracted common themes by consensus. Similarly, data regarding MAID outcomes and complications were summarised by identifying keywords (eg, 'IV access' or 'time to death') and from there, descriptive statistics were generated regarding the frequency with which various complications were identified in the literature.

\section{RESULTS}

\section{Selection of sources of evidence}

The initial online database search identified 12514 potential reports, and 22 additional reports were identified through the grey literature search (figure 1). After removing duplicate items, 11470 abstracts were screened, 582 of which met initial eligibility criteria and were assessed through full-text screening. Among these, articles were removed if they were of an ineligible reference type, reported on an ineligible population, only addressed MAID eligibility rather than provision, could not be successfully accessed or were one of the multiple reports on the same data. After applying these exclusion criteria, 163 articles were included in the review (see the online supplementary file 3).

\section{Characteristics of sources of evidence}

The identified reports were published between 1989 and 2019, with the greatest number published in 2010 $(n=14)$ and $2016(n=15)$, and $50 \%$ of reports published in 2009 or later. Report types included non-systematic reviews (including policy and legal reviews) ( $n=53)$, crosssectional surveys $(\mathrm{n}=32)$, MAID medication protocols $(n=19)$, cohort studies $(n=22)$ and cross-sectional studies, including death certificate studies $(n=14)$, qualitative studies $(n=13)$, clinical practice guidelines/best practices $(n=6)$ and systematic reviews $(n=2)$ (table 2). Reports described MAID provision in the Netherlands $(n=45)$, USA ( $n=43)$, Belgium $(n=29)$, Canada $(n=22)$, Switzerland $(n=8)$ or multiple regions $(n=13)$. For a complete list of data charted from each source of evidence, see the online supplementary file 4 .

\section{Synthesis of results Medications}

Close to half of the reports provided details for MAID administered by intravenous medications (75/163). A sample protocol for MAID administration by intravenous medication is presented in figure 2 and the frequencies and doses encountered for intravenous medications are shown in table 3 . The use of a general anaesthetic in combination with a neuromuscular blocker (NMB) was described in $57 \%$ of these studies $(43 / 75)$. The general anaesthetic mentioned was most commonly a barbiturate (34/43) or propofol (22/43). Neuromuscular blocking 


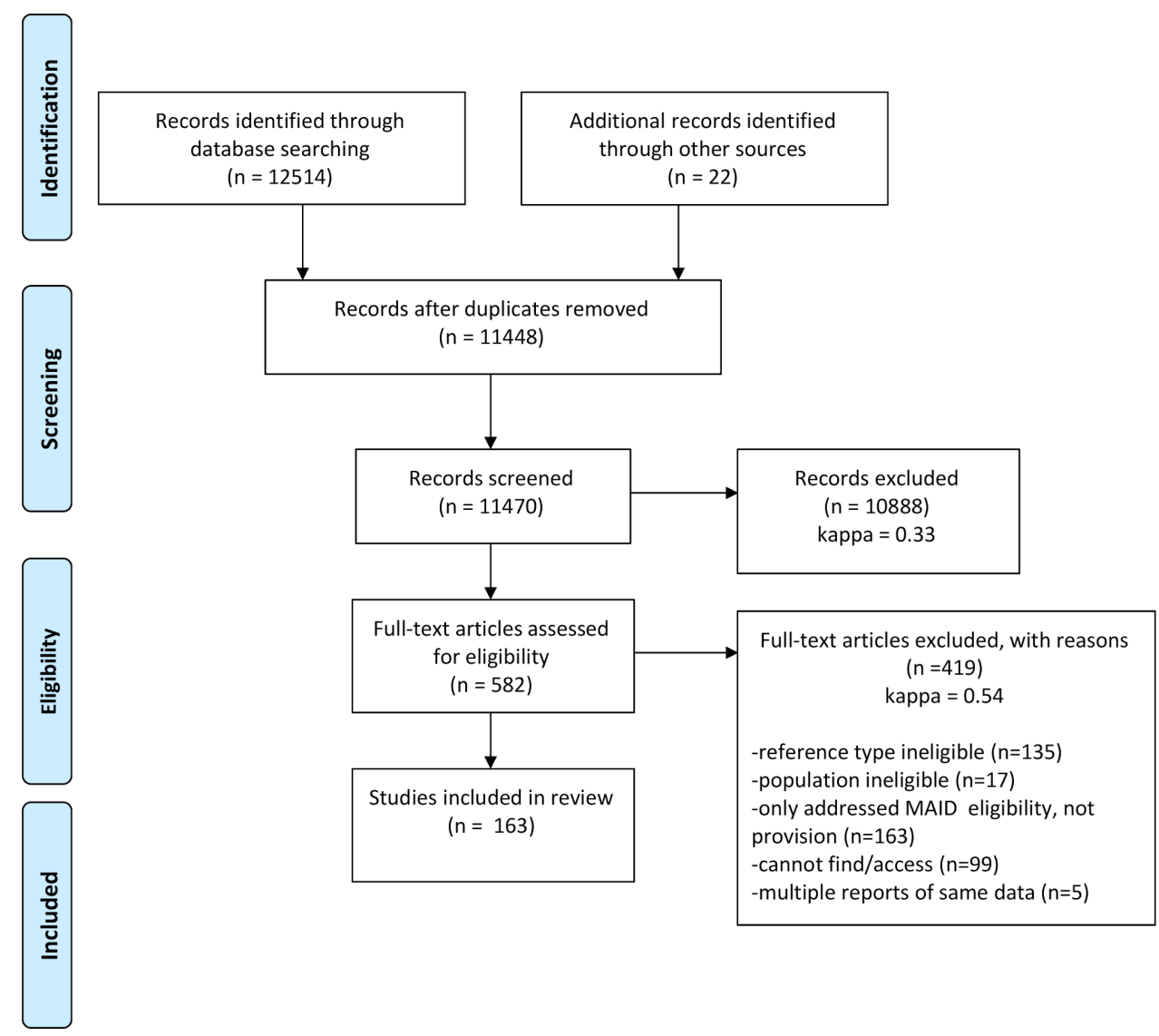

Figure 1 Preferred Reporting Items for Systematic Reviews and Meta-Analyses study selection flowchart. Adapted from Moher et al. ${ }^{14}$ MAID, medical assistance in dying.

agents most commonly used were cisatracurium, rocuronium and pancuronium. Of the 75 reports discussing intravenous protocols, 29 referred to the use of an anxiolytic prior to medication administration. Only two directly cardiotoxic agents were reported, bupivacaine $(2 / 75)$ and potassium chloride $(2 / 75)$.

Oral MAID regimes were detailed in 50 of 163 reports. A sample protocol for oral administration is presented in figure 3 , and the frequencies and doses for oral medications are presented in table 4. Barbiturate medications are mentioned in $94 \%$ of oral protocols $(47 / 50)$. The lifeending drug was a barbiturate alone in $74 \%(37 / 50)$ of oral regime studies, though barbiturates were also occasionally used with an opioid medication $(14 \%, 7 / 50)$ or alcohol $(6 \%, 3 / 50)$. Pentobarbital and secobarbital were the oral barbiturates most commonly mentioned, each referred to in $34 \%(17 / 50)$ of studies. Additionally, barbiturates were mentioned without specific medications or doses in 34\% (17/50) of reports. A single report described a combination of propranolol, digoxin and diazepam. To avoid vomiting, antiemetics, most commonly metoclopramide $(7 / 50)$ or ondansetron $(5 / 50)$, were given prior to administration of life-ending drugs was included in $36 \%$ of oral reports $(18 / 50)$. Anxiolytic medication such as midazolam or lorazepam appears in $12 \%(6 / 50)$ of studies. An 'as-needed' intravenous NMB was described as a backup in the case of failure of oral medications in
$26 \%(13 / 50)$ of reports. A single report described the use of helium gas to induce unconsciousness and death.

\section{Locations where assisted dying takes place}

Out of 163 articles, 65 described the setting for MAID administration. The two most common locations for MAID provision were in the hospital $(43 / 65)$ and at the patient's home $(43 / 65)$. Other settings include nursing home $(24 / 65)$, hospice $(7 / 65)$ and other settings $(7 / 65)$, including locations such as the headquarters of the nongovernmental organisation Dignitas in Switzerland.

\section{The role of health professionals in assisted dying}

The three health professions whose roles in MAID provision were most often described were physicians $(106 / 163)$, nurses $(33 / 163)$ and pharmacists $(32 / 163)$. Common roles described for physicians included prescribing $(83 / 106)$ and administering (75/106) medications, being present at death $(24 / 106)$ and pronouncing death $(12 / 106)$. The role of nurses was most often to administer medication $(17 / 35)$, support the patient $(16 / 35)$, prepare the route of administration $(13 / 35)$ and prepare medications $(6 / 35)$. Pharmacists' involvement was mainly to dispense medication $(34 / 35)$ and also included educating patients regarding the dispensed drugs $(12 / 35)$ and securing unused drugs $(7 / 35)$. Certain studies also discussed the involvement of 
Table 2 Report setting, study design and type of MAID protocol

\begin{tabular}{lc}
\hline Country of study & $\begin{array}{c}\text { Number (\% of } \\
\text { total studies) }\end{array}$ \\
\hline The Netherlands & $44(27.0)$ \\
\hline USA & $43(26.4)$ \\
\hline Belgium & $27(16.6)$ \\
\hline Canada & $22(13.5)$ \\
\hline Multiregion & $14(8.6)$ \\
\hline Switzerland & $8(4.9)$ \\
\hline Other & $5(3.1)$ \\
\hline Report type & \\
\hline Non-systematic review & $53(32.5)$ \\
\hline Survey & $32(19.6)$ \\
\hline MAID protocol & $19(11.7)$ \\
\hline Cohort study (retrospective) & $22(13.5)$ \\
\hline Cross-sectional (including death certificates) & $13(8.0)$ \\
\hline Qualitative study & $13(8.0)$ \\
\hline Clinical practice guideline/manual/handbook & $5(3.1)$ \\
\hline Systematic review & $2(1.2)$ \\
\hline Other & $4(2.5)$ \\
\hline Intravenous & \\
\hline Oral & $75(46)$ \\
\hline
\end{tabular}

MAID, medical assistance in dying.

other individuals, such as non-governmental organization (NGO) volunteers (Switzerland), other allied health, such as child life specialists, designated MAID coordinators and palliative care consultants. Finally, the role of family members was occasionally described (21 studies) and included supporting the patient $(17 / 21)$, retrieving medications (4/21) and assisting the preparation or administration oral life-ending medications (3/21).

\section{Outcomes and complications of assisted dying}

Of the 163 reports found, 40 described outcomes and complications in MAID provision. For intravenous administration $(n=22)$, complications included difficulty in obtaining or maintaining intravenous access $(4 / 22)$, the patient dying too slowly or not dying $(6 / 22)$, patient dying too quickly $(3 / 22)$, difficulty in pushing a large syringe, pain on injection, need for a backup kit and inappropriate drugs given (1/22 each). For oral administration $(\mathrm{n}=17)$, complications included prolonged duration of the dying process $(13 / 17)$, vomiting $(6 / 17)$, myoclonus/ seizures $(2 / 17)$, poor taste of the cocktail and the need for intravenous backup $(1 / 17)$. One study describing the inhalation route described moor mask fit problems.

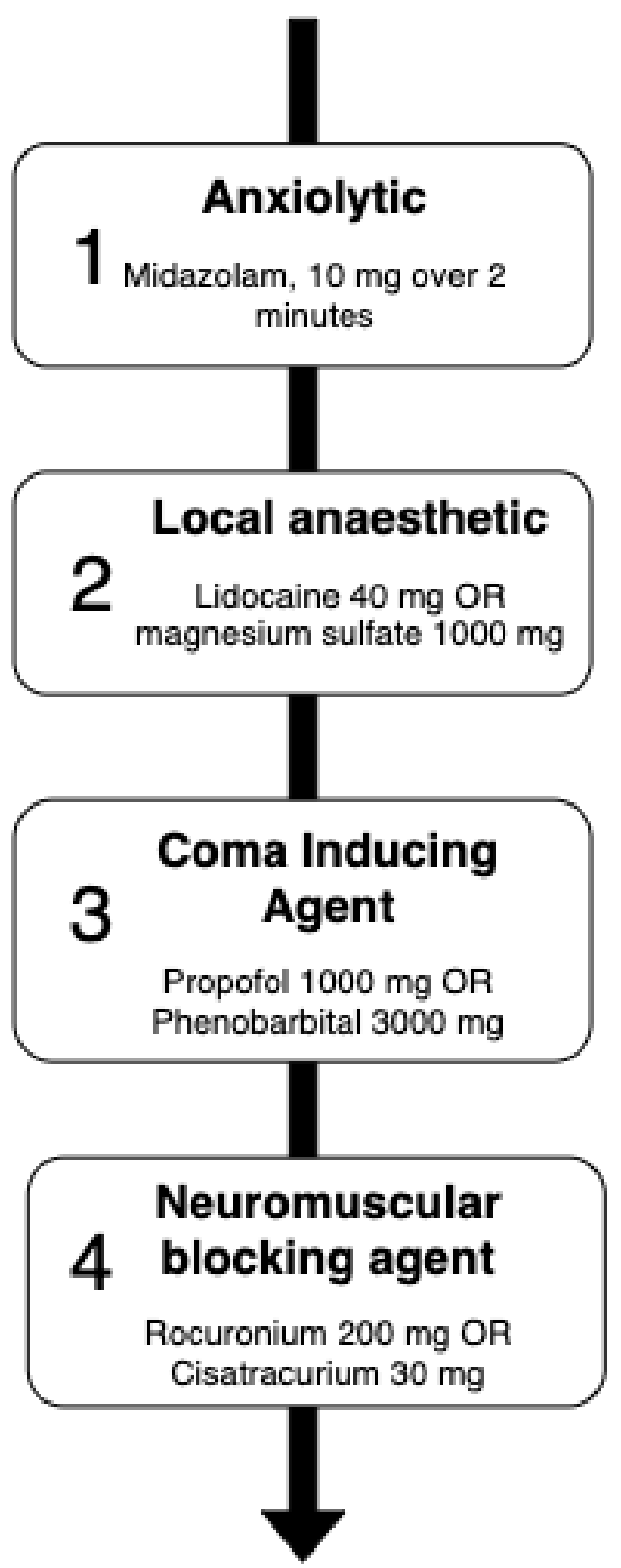

Figure 2 Sample protocols for MAID administration by intravenous medications, including medications and dose ranges encountered in the scoping review. MAID, medical assistance in dying.

\section{DISCUSSION}

\section{Summary of evidence}

We found 163 published and unpublished reports describing the provision of MAID, which varied greatly in geographic origin, report type and items reported. The content of the reports was correspondingly diverse, with a wide variety of medications used for both intravenous and oral routes. Intravenous drugs were usually given in a sequence, with an anxiolytic (most commonly midazolam), followed by a sedative/anaesthetic (with or without an opioid) followed by a NMB. Direct cardiotoxic medications (eg, potassium and bupivacaine) were used infrequently, despite the fact that these would be expected to result in a rapid, painless death very shortly after injection. There are several possible reasons for this. First, providers 
Table 3 Medication, doses and frequency encountered for MAID provision by intravenous medication

\begin{tabular}{|c|c|c|}
\hline Description & Dose range & Frequency \\
\hline \multicolumn{3}{|l|}{ Benzodiazepines } \\
\hline $\begin{array}{l}\text { Benzodiazepine not } \\
\text { specified }\end{array}$ & PRN & 14 \\
\hline Diazepam & $10-120 \mathrm{mg}$ & 3 \\
\hline Lorazepam & $2.5-5 \mathrm{mg}$ PRN & 2 \\
\hline Midazolam & 2-120mg, PRN & 30 \\
\hline \multicolumn{3}{|l|}{ Other sedatives } \\
\hline Propofol & 1000-2000 mg, PRN & 21 \\
\hline Pentobarbital & $1-15 g$ & 7 \\
\hline Thiopental & $1-2 \mathrm{~g}, 20 \mathrm{mg} / \mathrm{kg}$ & 21 \\
\hline Secobarbital & $9 g$ & 5 \\
\hline Phenobarbital & $3000 \mathrm{mg}$ & 8 \\
\hline Vesparax & Not reported & 1 \\
\hline Chloral hydrate & $35-40 \mathrm{mg}$ & 1 \\
\hline \multicolumn{3}{|c|}{ Neuromuscular blockers } \\
\hline $\begin{array}{l}\text { Neuromuscular } \\
\text { blocker not specified }\end{array}$ & PRN & 26 \\
\hline Mivacurium & Not reported & 1 \\
\hline Atracurium & $50-100 \mathrm{mg}$ & 2 \\
\hline Alcuronium & $45 \mathrm{~g}$ & 1 \\
\hline Pancuronium, PRN & $18-20 \mathrm{mg}$ & 9 \\
\hline Rocuronium & 50-300 mg, PRN & 17 \\
\hline Cisatracurium & $30-40 \mathrm{mg}$ & 7 \\
\hline Vecuronium & $10-60 \mathrm{mg}$ & 6 \\
\hline Curare & Not reported & 3 \\
\hline \multicolumn{3}{|l|}{ Opioids } \\
\hline Opioids NOS & NA & 20 \\
\hline Morphine & $16-480 \mathrm{mg}$ & 3 \\
\hline Fentanyl & $25-1500 \mu g$ & 2 \\
\hline \multicolumn{3}{|l|}{ Cardiotoxic agents } \\
\hline Potassium chloride & Not reported & 3 \\
\hline Bupivacaine & $400 \mathrm{mg}$ & 2 \\
\hline \multicolumn{3}{|l|}{ Local anaesthetics } \\
\hline Lidocaine & $40-120 \mathrm{mg}$ & 20 \\
\hline Magnesium sulfate & $1000 \mathrm{mg}$ & 5 \\
\hline
\end{tabular}

MAID, medical assistance in dying; NOS, not otherwise specified; PRN, pro re nata ("as needed").

may be unfamiliar with and thus reluctant to use these agents, as outside of MAID, clinicians rarely administer drugs that are designed to stop a patient's heart. Second, anticipated discomfort of providers and families with immediate death-'death happened too quickly' was described as a complication in three reports, indicating that even with a planned rapid assisted death, people still expect there to be a 'process' of dying after medications are administered. Third, it may be that MAID providers

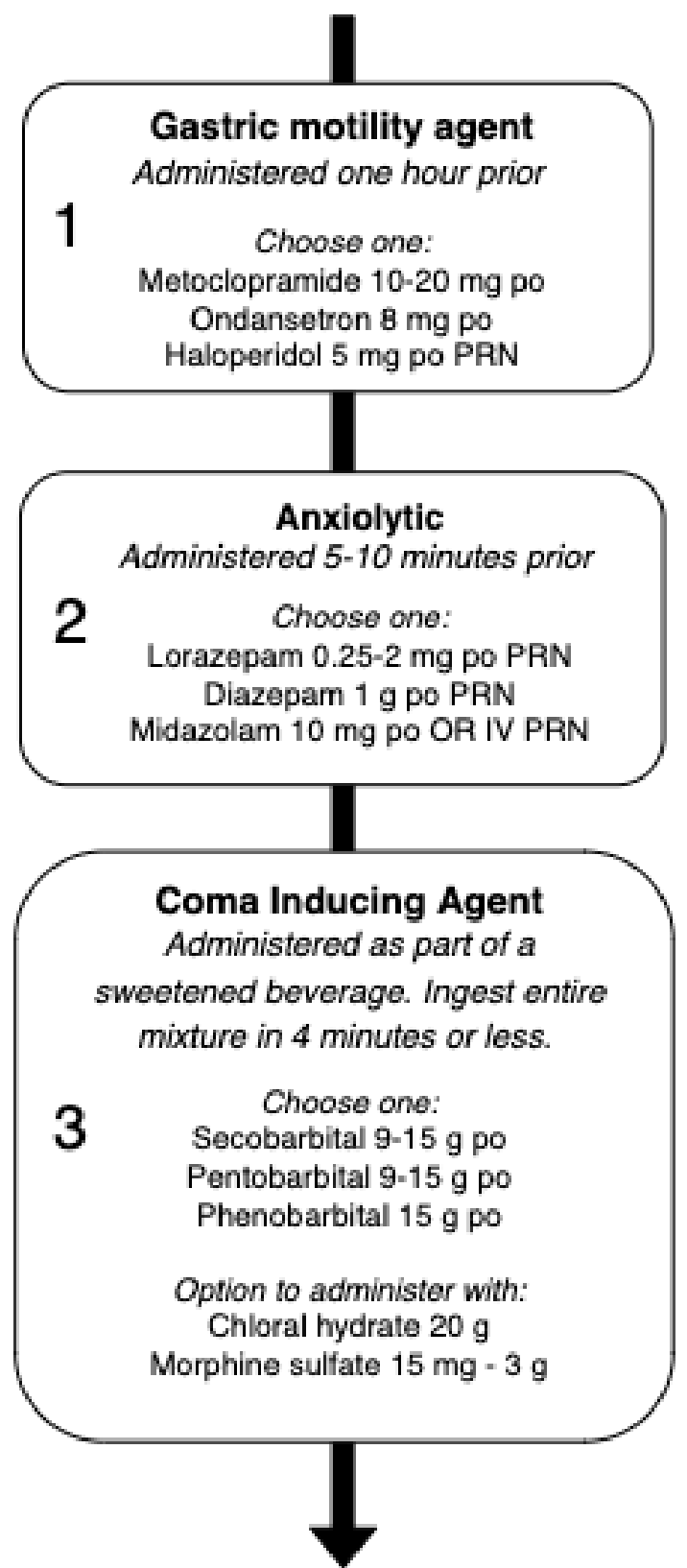

Figure 3 Sample protocols for MAID administration via oral medications, including medications and dose ranges encountered in the scoping review. MAID, medical assistance in dying.

are uncomfortable with the directness of injecting medication and stopping the patient's heart. Administering a NMB and waiting for a patient to die of $\mathrm{CO}_{2}$ narcosis or hypoxia maintains some element of 'indirectness' to the patient's death. Finally, these medications may be avoided simply because it is not required to directly stop the heart in the presence of deep sedation and anoxiathus cardiotoxic agents are seen as unnecessary.

The reports we found did not generally link data between medications, locations, providers and outcomes. As a result it is not possible to determine which medications or combinations of medications are most effective and result in the fewest complications and least distress for patients, providers and families. However, for providers 
Table 4 Medication, doses and frequency encountered for MAID provision by oral medication.

\begin{tabular}{|c|c|c|}
\hline Description & Dose range & Frequency \\
\hline \multicolumn{3}{|l|}{ Barbiturates } \\
\hline Barbiturate not specified & NA & 17 \\
\hline Pentobarbital & $9-15 \mathrm{~g}$ & 21 \\
\hline Phenobarbital & $20 \mathrm{~g}$ & 10 \\
\hline Secobarbital & $9-15 \mathrm{~g}$ & 20 \\
\hline Brallobarbitalum & Not reported & 1 \\
\hline Sodium thiopental & Not reported & 1 \\
\hline \multicolumn{3}{|l|}{ Benzodiazepines } \\
\hline $\begin{array}{l}\text { Benzodiazepine not } \\
\text { specified }\end{array}$ & NA & 6 \\
\hline Diazepam & $1 \mathrm{~g}$ & 3 \\
\hline Lorazepam & $0.25-2 \mathrm{mg}$ PRN, IV & 3 \\
\hline Midazolam & $10 \mathrm{mg}, \mathrm{PRN}, \mathrm{IV}$ & 2 \\
\hline \multicolumn{3}{|l|}{ Antiemetics } \\
\hline Antiemetic not specified & NA & 8 \\
\hline Metoclopramide & $10-20 \mathrm{mg}$ & 8 \\
\hline Ondansetron & $8 \mathrm{mg}$ & 5 \\
\hline Haloperidol & $5 \mathrm{mg}, \mathrm{PRN}$ & 2 \\
\hline \multicolumn{3}{|l|}{ Miscellaneous sedatives } \\
\hline Chloral hydrate & $20 \mathrm{~g}$ & 5 \\
\hline \multicolumn{3}{|l|}{ Cardiotoxic agents } \\
\hline Digoxin & $50 \mathrm{mg}$ & 3 \\
\hline Propranolol & $2 \mathrm{~g}$ & 3 \\
\hline \multicolumn{3}{|l|}{ Opioids } \\
\hline Morphine & $15 \mathrm{mg}-3 \mathrm{~g}$ & 13 \\
\hline Dextropropoxyphene & Not reported & 2 \\
\hline \multicolumn{3}{|c|}{ Neuromuscular blocker (for IV backup use) } \\
\hline Neuromuscular blocker & IV, PRN (backup) & 11 \\
\hline
\end{tabular}

IV, intravenous; MAID, medical assistance in dying; PRN, pro re nata ("as needed").

and healthcare organisations that provide assisted dying, our scoping review does provide an overview of what the most commonly described practices are, worldwide. There is a need for future research in this area, including understanding patient and family perspectives of what makes a 'good' assisted death; descriptions of which complications are most burdensome to patients, families and providers; consistent definitions and outcome reporting practices of MAID provision; and comprehensive, prospective data collection of clinical practice. Taken together, this information would allow comparative research between different approaches to MAID and allow clinical researchers to identify the medications, dosages, and administration techniques and procedures that are cost-effective, simple to administer and mitigate distress for those involved.

\section{Strengths}

Strengths of our scoping review included its very broad and inclusive search strategy, screening in duplicate by two investigators at both the title/abstract and fulltext levels. As well, we used a prepublished protocol that allowed for a peer review and input prior to study completion and to ensure that our very broad review accomplished and reported its stated objectives and outcomes.

\section{Limitations}

While we described a wide variety of methods for providing MAID, few reports described the number of times the protocol has been used. Similarly, there are likely to be differences between what is written in a protocol and what is actually done in practice. It also does not capture practices which are not formally recorded, either as a publication or as a policy or procedure. As a result, our review cannot provide insight into which approaches to providing aid in dying are most commonly used but only those which are most commonly described in written form. As well, policies and protocols from older reports may have changed since their first publication in the medical literature.

Our study is also limited by its emphasis on Canadian practice. As most of this review's authors are Canadian, we were able to gather a larger number of policies and protocols from Canada, despite vigorous attempts to obtain them from other jurisdictions. The comparatively small number of protocols from other countries may be related to the development of regional standardised approaches to MAID provision (eg, the National Dutch Protocol) resulting in a smaller total number of policies and protocols and due to a paucity of English-language protocols and policies. Of note, the Canadian policies and protocols are more recent than those in other countries (eg, the Netherlands, Belgium, Luxembourg and the USA), generally dating back to the passage of Bill C-14 in June 2016. Canadian policy and practice are likely to undergo further changes as more experience with MAID is accrued, potentially limiting our report's validity as a description of current practice. Reassuringly, we have informally reviewed a sample of more recent Canadian MAID protocols and found there to be little difference. Data from the Fourth Interim Report on MAID suggest that to date, the vast majority of assisted deaths in Canada continue to use the intravenous route. ${ }^{2}$

\section{CONCLUSIONS}

We described the published and unpublished literature on MAID provision including common medications and doses, roles of healthcare professionals and families, and complications that may cause distress. Future research should aim to identify the medications, dosages, and administration techniques and procedures, which 
produce the most predictable outcomes and mitigate distress for those involved.

\section{Author affiliations}

${ }^{1}$ Department of Emergency Medicine, Faculty of Medicine, University of Ottawa, Ottawa, Ontario, Canada

${ }^{2}$ Department of Medicine, McMaster University, Hamilton, Ontario, Canada

${ }^{3}$ Division of Critical Care Medicine, Western University, London, Ontario, Canada

${ }^{4}$ Hamilton Health Sciences, Hamilton, Ontario, Canada

${ }^{5}$ Department of Clinical and Organizational Ethics, The Ottawa Hospital, Ottawa, Ontario, Canada

${ }^{6}$ Division of Emergency Medicine, Department of Medicine, Hamilton Health Sciences, Hamilton, Ontario, Canada

${ }^{7}$ Department for Primary and Community Care, Radboudumc, Nijmegen, The Netherlands

${ }^{8}$ Department of Health Research Methods, Evidence, and Impact, McMaster University, Hamilton, Ontario, Canada

Twitter lan Ball @Ball and Simon 0czkowski @Simon_0cz

Acknowledgements The authors thank the numerous MAID providers and healthcare organisations that provided access to their policies and protocols. They also thank Laura Banfield for providing assistance with the electronic search strategies.

Contributors MZ assisted with data acquisition and interpretation, manuscript drafting and revision. CS assisted with conception of the study, data acquisition and revision. IB, GK, AC, MK, PQM, MD and AF assisted with study conception, design and manuscript revision. SO assisted with study conception, design, data acquisition, drafting and revision.

Funding This research is supported by a Hamilton Academic Health Sciences Organisation's innovation fund. S0 is supported by an internal career award from the Department of Medicine at McMaster University. MZ is supported by the McMaster Medical Student Research Excellence Scholarship (MAC RES).

Competing interests None declared.

Patient and public involvement Patients and/or the public were not involved in the design, or conduct, or reporting, or dissemination plans of this research.

Patient consent for publication Not required.

Provenance and peer review Not commissioned; externally peer reviewed.

Data availability statement The majority of data relevant to this study are included in the article or uploaded as supplemental information. Additional data are available upon request.

Open access This is an open access article distributed in accordance with the Creative Commons Attribution Non Commercial (CC BY-NC 4.0) license, which permits others to distribute, remix, adapt, build upon this work non-commercially, and license their derivative works on different terms, provided the original work is properly cited, appropriate credit is given, any changes made indicated, and the use is non-commercial. See: http://creativecommons.org/licenses/by-nc/4.0/.

ORCID iDs

Max Zworth http://orcid.org/0000-0002-9120-998X

Simon 0czkowski http://orcid.org/0000-0002-2874-8948

\section{REFERENCES}

1 First Session of the Parliament of Canada. Bill C-14: an act to amend the criminal code and to make related amendments to other acts (medical assistance in dying); 2016.

2 Health Canada. Fourth interim report on medical assistance in dying in Canada, 2019.

3 Nicol J. Medical assistance in dying: the law in selected Jurisdictions outside Canada, 2019.

4 Harty C, Chaput AJ, Trouton K, et al. Oral medical assistance in dying (MAiD): informing practice to enhance utilization in Canada. Can J Anaesth 2019;66:1106-12.

5 Hedberg K, New C. Oregon's death with dignity act: 20 years of experience to inform the debate. Ann Intern Med 2017;167:579.

6 Hendin H, Rutenfrans C, Zylicz Z. Physician-Assisted suicide and euthanasia in the Netherlands. lessons from the Dutch. JAMA 1997;277:1720-2.

7 Groenewoud JH, van der Heide A, Onwuteaka-Philipsen BD, et al. Clinical problems with the performance of euthanasia and physicianassisted suicide in the Netherlands. N Engl J Med 2000;342:551-6.

8 Chin AE, Hedberg K, Higginson GK, et al. Legalized physicianassisted suicide in Oregon - the first year's experience. $N$ Engl J Med Overseas Ed 1999;340:577-83.

9 Sullivan AD, Hedberg K, Fleming DW. Legalized physicianassisted suicide in Oregon--the second year. $N$ Engl J Med 2000;342:598-604.

10 Ganzini L, Nelson HD, Schmidt TA, et al. Physicians' experiences with the Oregon death with dignity act. $N$ Engl J Med 2000;342:557-63.

11 Government of Canada. Regulations for the monitoring of medical assistance in dying: SOR/2018-166. Canada Gazette, part II. 152 , 2018.

12 Joanna Briggs Institute. The Joanna Briggs institute reviewers' manual 2015: Methodology for JBI scoping reviews. Australia: Joanna Briggs Institute, 2015.

13 Oczkowski SJW, Ball I, Saleh C, et al. The provision of medical assistance in dying: protocol for a scoping review. BMJ Open 2017;7:e017888.

14 Moher D, Liberati A, Tetzlaff J, et al. Preferred reporting items for systematic reviews and meta-analyses: the PRISMA statement. PLoS Med 2009;6:e1000097. 